\title{
New splicing pathogenic variant in EBP causing extreme familial variability of Conradi-Hünermann-Happle Syndrome
}

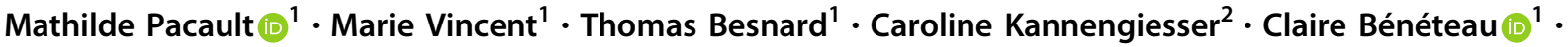 \\ Sébastien Barbarot ${ }^{3} \cdot$ Xénia Latypova $^{1} \cdot$ Khaldia Belabbas $^{4} \cdot$ Antonin Lamazière $^{5} \cdot$ Norbert Winer $^{6}$. \\ Madeleine Joubert ${ }^{7}$. Stéphane Bézieau $\left(10^{1} \cdot\right.$ Bertrand Isidor $^{1} \cdot$ Sandra Mercier $^{1} \cdot$ Mathilde Nizon $^{1}$. \\ Stéphanie Leclerc-Mercier ${ }^{8} \cdot$ Smail Hadj-Rabia $^{8} \cdot$ Fabienne Dufernez $^{9}$
}

Received: 7 February 2018 / Revised: 20 June 2018 / Accepted: 26 June 2018 / Published online: 22 August 2018

(c) European Society of Human Genetics 2018

\begin{abstract}
$\mathrm{X}$-linked dominant chondrodysplasia punctata (CDPX2 or Conradi-Hünermann-Happle syndrome, MIM \#302960) is caused by mutations in the $E B P$ gene. Affected female patients present with Blaschkolinear ichthyosis, coarse hair or alopecia, short stature, and normal psychomotor development. The disease is usually lethal in boys. Nevertheless, few male patients have been reported; they carry a somatic mosaicism in $E B P$ or present with Klinefelter syndrome. Here, we report CDPX2 patients belonging to a three-generation family, carrying the splice variant c. $301+5 \mathrm{G}>\mathrm{C}$ in intron 2 of $E B P$. The grandfather carries the variant as mosaic state and presents with short stature and mild ichthyosis. The mother also presents with short stature and mild ichthyosis and the female fetus with severe limb and vertebrae abnormalities and no skin lesions, with random $\mathrm{X}$ inactivation in both. This further characterizes the phenotypical spectrum of CDPX2, as well as intrafamilial variability, and raises the question of differential EBP mRNA splicing between the different target tissues.
\end{abstract}

These authors contributed equally: Mathilde Pacault, Marie Vincent.

Electronic supplementary material The online version of this article (https://doi.org/10.1038/s41431-018-0217-0) contains supplementary material, which is available to authorized users.

\section{Marie Vincent}

marie.vincent@chu-nantes.fr

1 Service de génétique médicale, CHU Nantes, Nantes, France

2 Département de génétique, Hôpital Bichat, AP-HP, Paris, France

3 Service de dermatologie, CHU Nantes, Nantes, France

4 Laboratoire Commun de Biologie et Génétique Moléculaires (LCBGM), Hôpital Saint Antoine, AP-HP, Paris, France

5 Plateforme de spectrométrie de masse (PM2), Hôpital SaintAntoine, APHP, ERL Inserm U1157, UMR 7203, Paris, France

6 Service de gynécologie-obstétrique, CHU Nantes, Nantes, France

7 Service d'anatomopathologie, CHU Nantes, Nantes, France

8 Department of Dermatology, Reference Center for Genodermatoses (MAGEC), Necker-Enfants Malades Hospital (AP-HP), Imagine Institute, Paris Descartes-Sorbonne Paris-Cité University, Paris, France

9 Service de génétique, CHU de Poitiers, Poitiers, France

\section{Introduction}

X-linked dominant chondrodysplasia punctata (CDPX2, MIM \#302960), also known as Conradi-HünermannHapple syndrome, is a rare heterogeneous disorder characterized by asymmetrical bone defects including epiphyseal stippling, shortening of limbs, kyphoscoliosis, neonatal ichthyosis with follicular atrophoderma later in life, patchy alopecia or coarse hair, cataracts, and craniofacial defects $[1,2]$. Affected patients usually present with short stature but normal psychomotor development. CDPX2 was initially described in females and is considered to be lethal early in pregnancy in hemizygous males. Most male patients described have carried somatic mosaicism [3, 4]. One patient with Klinefelter syndrome has also been reported [5]. High clinical variability has been reported in females, from severe prenatally diagnosed cases to mildly affected adults with a phenotype limited to skin, suggested to be caused by skewed X-inactivation [6, 7] or somatic mosaicism [8].

CDPX2 is caused by mutations in the EBP gene (chromosome Xp11.23) encoding the emopamil-binding protein, which plays a key role in the last steps of cholesterol biosynthesis [9, 10]. Diagnosis is suspected in patients 
Fig. 1 Pedigree of the family with the different phenotypic features. III.1-Proband: $24 \mathrm{WG}$ fetus, presenting with vertebral abnormalities and spinal puzzle, narrow thorax, chondral punctuations, and asymmetrical shortening of long bones. II.2Mother: short stature, alopecia, coarse hair, xerosis. I.1Grandfather: short stature, alopecia, coarse hair, ichthyosis, and asymmetrical length of the legs. Sanger analysis of $E B P$ intron 2 showing the c. $301+5$ $\mathrm{G}>\mathrm{C}$ variant at heterozygous state in the proband (III.1) and her mother (II.2), and at a mosaic state in the grandfather (I.1)

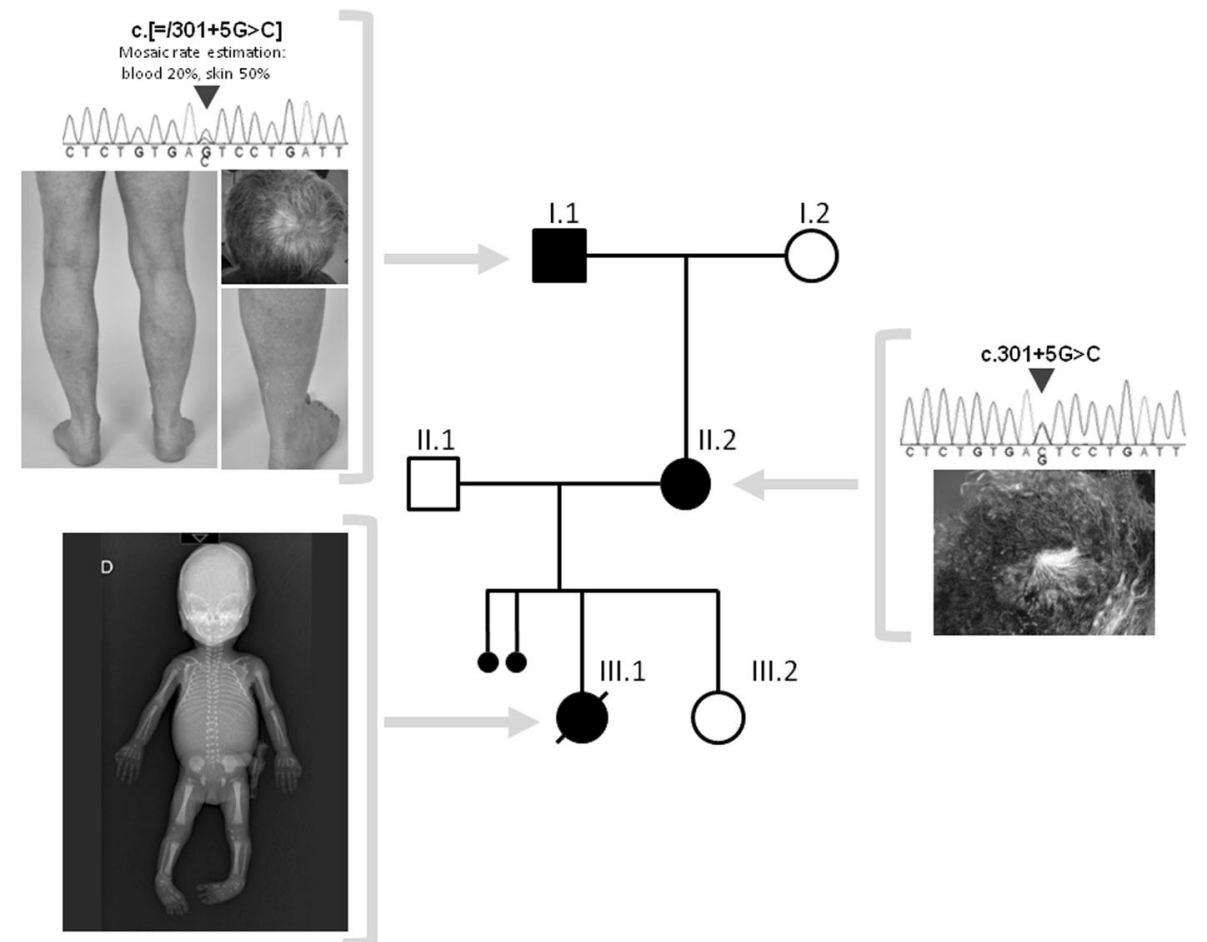

exhibiting elevated levels of cholesterol precursors in plasma and involved tissues. Sequencing EBP gene confirms the diagnosis. To date, more than $70 \mathrm{EBP}$ mutations have been reported. Genotype to phenotype correlation remains uncertain [11].

Here, we report the observation of a severely affected female fetus revealing a family history of CDPX2 with a mildly affected mother and grandfather.

\section{Patients and methods}

\section{Patients}

Patient II.2, a 22 weeks of gestation (WG) pregnant woman, was referred for fetal bone anomalies detected on the second ultrasound examination of her uneventful pregnancy (Fig. 1). The fetus (III.1) presented with severe shortening of long bones of upper and lower limbs, multiple epiphyseal stippling and vertebral abnormalities with spinal puzzle. Given the diagnosis of severe punctuated chondrodysplasia, the functional risk to the child and the initial hypothesis of peroxysomal disease, the pregnancy was terminated at 24 WG. Fetal autopsy revealed an asymmetrical rhizomelic shortening of the limbs without skin abnormalities, visceral malformation, or facial dysmorphism. Skeletal X-rays showed multiple and symmetrical bone punctuations of the tarsus, the carpus, the ribs, the knee-caps, and the spine, associated to vertebral puzzle (Fig. 1). The karyotype (46, $\mathrm{XX}$ ) was normal. Peroxysomal disease has been excluded after the termination with normal very long chain fatty acids on fetal plasma and normal DHAP-AT on cultured amniocytes. The diagnosis of CDPX2 was confirmed on fetal tissue.

Patient II. 2 presented with short stature $(150 \mathrm{~cm})$, xerosis, patchy alopecia of the scalp and coarse hair. Musculoskeletal examination was normal (Fig. 1).

Family history showed that Patient I-1 presented with low height $(165 \mathrm{~cm})$, lower limb asymmetry, fluctuating asymmetrical and linear ichthyosis associated to two patches of scalp alopecia (Fig. 1).

Informed consent was obtained from both patients. Genomic DNA was extracted from peripheral blood for individual II.2, from peripheral blood and skin biopsy for individual I.1, and from fetal lung for the fetus (III.1).

\section{Sterol analysis}

Cholesterol, 7 dehydrocholesterol (7DHC; cholest-7-en-3 $\beta$ ol), lathosterol, epicoprostanol ( $5 \alpha$-cholestan-3 $\beta$-ol) and desmosterol (cholesta-5,24-dien-3 $\beta$-ol) were obtained from Sigma (Saint-Louis, MO, USA). Solvents of the analytical grade were purchased from Sigma. The silylation reagent (Regisil) was obtained from Interchim (Montluçon, France).

A volume of $200 \mu \mathrm{l}$ of fresh plasma was mixed in $5 \mathrm{ml}$ of the solvent mixture chloroform/methanol $2 / 1$ (v/v) containing the internal standard (epicoprostanol). Lipids were partitioned in chloroform after addition of saline and saponified by methanolic potassium hydroxide $(0.5 \mathrm{~N}$, $\left.60{ }^{\circ} \mathrm{C}, 15 \mathrm{~min}\right)$. Released fatty acids were methylated by 


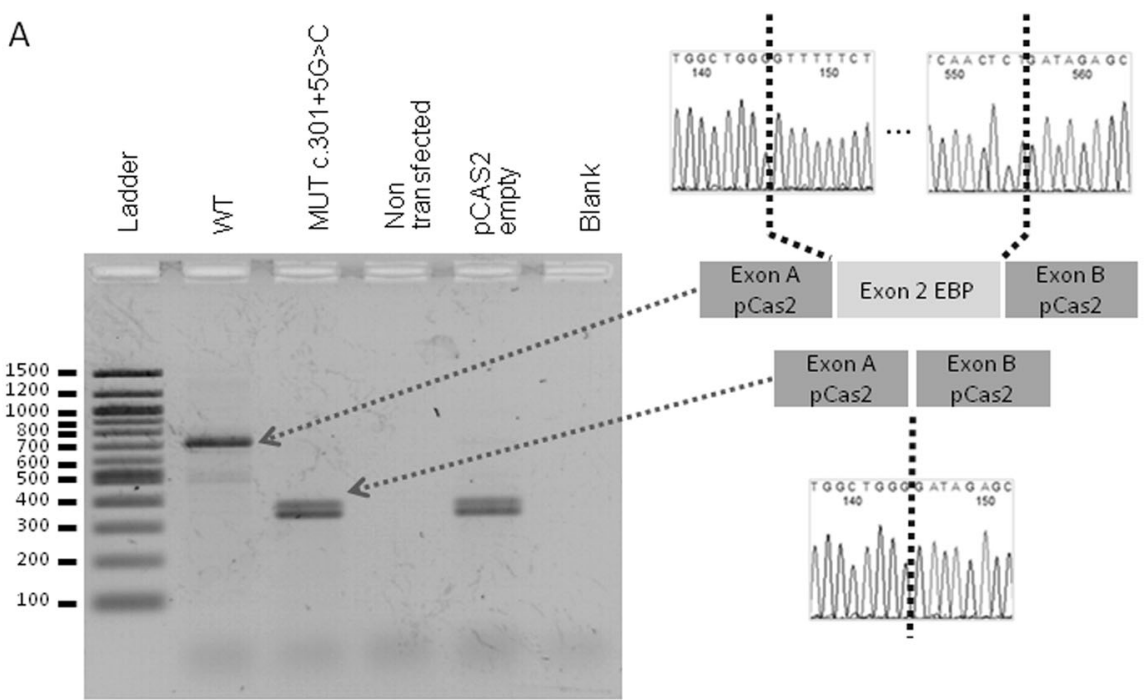

B

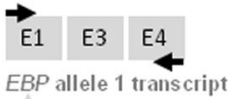

EBP allele 1 transcript
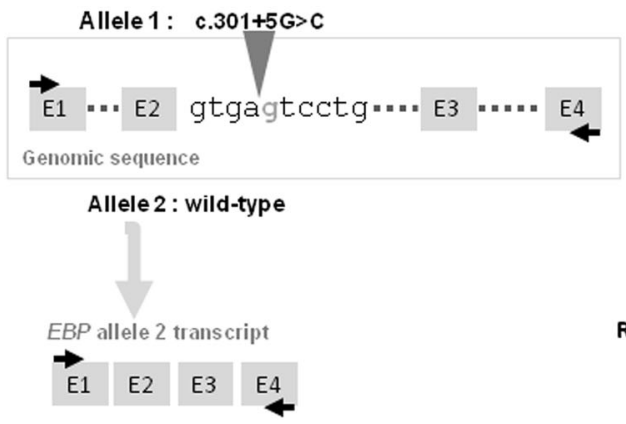

FORWARD

REVERSE

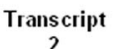

Fig. 2 Molecular characterization of the splicing defect EBP variant. a Ex vivo analysis of the c. $301+5 \mathrm{G}>\mathrm{C}$ variant in EBP. Gel electrophoresis of RT-PCR products obtained from the minigene assay. Sanger sequencing resulting from the normal and the mutant splice site are represented. b RT-PCR products analysis obtained from the fetal tissue. Left: schematic representation of the genomic sequence

BF3-methanol $\left(12 \%, 60{ }^{\circ} \mathrm{C}, 15 \mathrm{~min}\right)$ to prevent interference with the chromatography of sterols. Sterols were reextracted in hexane and silylated as described previously [12]. The trimethylsilylether derivatives of the sterols were separated by gas liquid chromatography (Hewlett-Packard 6890 Series) in a medium polarity capillary column provided by Restesk (Evry, France) (RTX-65, (65\% diphenyl $35 \%$ dimethyl polysiloxane), length $30 \mathrm{~m}$, diameter 0.32 $\mathrm{mm}$, film thickness $0.25 \mu \mathrm{m}$ ). Sterols were identified by comparison of the mass spectra with the NIST library. The mass spectrometer (Agilent 5875 inert XL Mass Selective Detector, Agilent Technologies France) in series with the
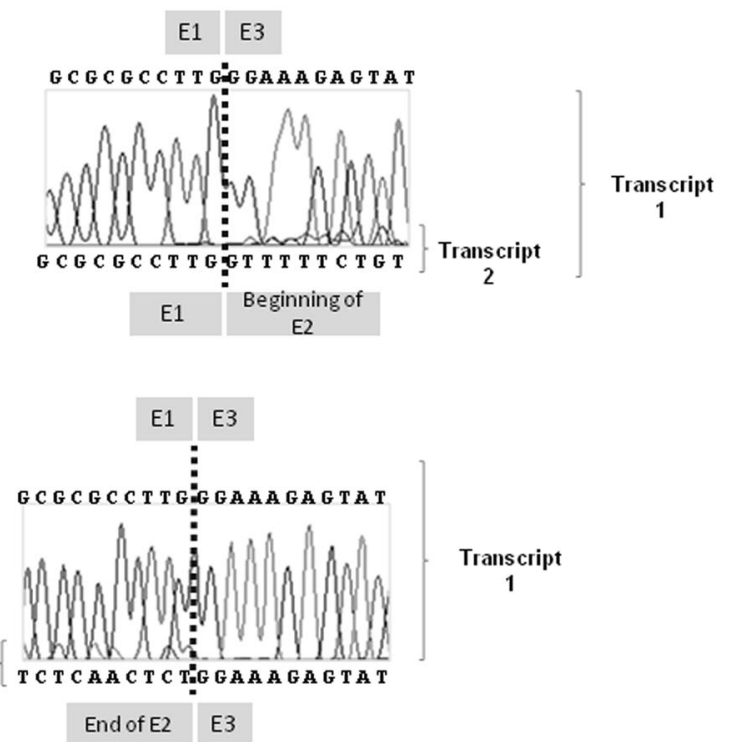

surrounding the EBP variant. Black arrows correspond to the positions of the primers used for RT-PCR and sequencing. Right: Forward and reverse Sanger sequencing of the RT-PCR products obtained from the patient corresponding to different transcripts (mutated transcript 1 with skipping of the exon 2 and longer normal transcript 2 less amplified)

GC was set up for the detection of the positive fragment ions. Fragment ions were produced in the electron impact mode at $70 \mathrm{eV}$. The sterols were identified in the scanning mode and quantified by selective monitoring of the prominent ions after normalization with the internal standard epicoprostanol and calibration with weighed standards.

\section{Molecular analysis}

Following informed consent for genetic testing, genomic DNA was extracted from peripheral blood leucocytes by a phase exchange method (Puregene, Qiagen) and quantified 
with Spectrophotometer Nanodrop (Shimadzu). The entire coding region of EBP gene and flanking intronic sequences were amplified by polymerase chain reaction (PCR) using previously described primers [3] with a modification for exon 2: 5'-TTCGGTCCATTTACATTTCTCA-3' (forward) to explore the intron/exon junction. Cycling conditions were as follows: 35 cycles at $95^{\circ} \mathrm{C}$ for $5 \mathrm{~min}, 95^{\circ} \mathrm{C}$ for $30 \mathrm{~s}$, $60{ }^{\circ} \mathrm{C}$ for $30 \mathrm{~s}$, and $72{ }^{\circ} \mathrm{C}$ for $45 \mathrm{~s}$ and extension at $72{ }^{\circ} \mathrm{C}$ for $7 \mathrm{~min}$. PCR products were sequenced for both strands on an automated sequencer (3500XL Dx, Applied Biosystems).

Results are reported with exons numbered according to NG_007452.1 accession.

\section{$X$ inactivation analysis}

Analysis of leukocyte $\mathrm{X}$ chromosome inactivation at the HUMARA locus was performed as previously described [13]. Briefly, genomic DNA was digested using the methylation-sensitive enzyme HhaI and HpaII. Undigested and digested DNA were then subjected to PCR amplification of the highly polymorphic CAG-repeat sequence in the human androgen receptor (HUMARA), with specific fluorescent primers. PCR products were analyzed on an automated DNA sequencer (ABI PRISM 3130 DNA sequencer, Life technologies). A control DNA with known skewed $\mathrm{X}$ inactivation was also tested in the same experiment.

\section{Splice-site predictions}

The predicted effect of the variant on RNA splicing was studied using the five tools combined in the splicing prediction module of the Alamut Visual Software (V2.7, Interactive Biosoftware, Rouen, France).

\section{Splicing minigene reporter assay}

The splicing minigene assay was adapted from the method described by Gaildrat [14]. Wild-type genomic segments corresponding to the exon 2 of EBP (NM_006579.2) with flanking intronic sequences (701 bp) were amplified by PCR from control genomic DNA (Q5 High-Fidelity DNA Polymerase, NEB). The primer sequences and PCR conditions used are available upon request. The PCR product was cloned into the pCAS2 vector digested with BamHI and $M l u \mathrm{I}$ enzymes. Plasmids were purified and sequenced on the inserted region. Mutant construct containing the c. $301+5$ $\mathrm{G}>\mathrm{C}$ variant was generated by using the Q5 site-directed mutagenesis kit (NEB) according to the manufacturer's instructions. Purified plasmids were sequenced to confirm the site-directed mutagenesis. Normal and mutant pCAS2 minigenes were transfected into HeLa cells using Fugene 6 transfection reagent (Promega) which were harvested $24 \mathrm{~h}$ later. Total RNA was isolated using the NucleoSpin RNA Plus kit (Macherey-Nagel). Oligo(dT) primers and the ProtoScript II First Strand cDNA Synthesis Kit (NEB) was used to synthesize cDNA, according to the manufacturer's instructions. RT-PCR was performed using primers located in exons A and B of the pCAS2 vector. The exons $\mathrm{A}$ et $\mathrm{B}$ refer to the pCAS2 vector as it is shown in Fig. 2a. Amplified products were visualized by GelRed staining (Biotium) after electrophoresis on $1 \%$ agarose gel and Sanger sequenced to describe splicing variations.

\section{Tissue mRNA analysis}

Total RNA was extracted from fetal lung using the NucleoSpin RNA Plus kit (Macherey-Nagel) and cDNA was generated following the instructions described above. The primer sequences and RT-PCR conditions used are available upon request. RT-PCR products were analyzed on $1 \%$ agarose gel electrophoresis and Sanger sequenced.

\section{Database submission}

Clinical data of fetus and gene variant in $E B P$ were submitted at www.LOVD.nl/EBP with ID 00155196.

\section{Results}

\section{Biochemical diagnosis}

Sterol analysis was performed on fetal tissue, and elevated hepatic 8-lathosterol and 8-dehydrocholesterol with a (8Lathosterol + 8-dehydrocholesterol)/cholesterol ratio of 0.0829 was found, consistent with a deficit in the last steps of cholesterol biosynthesis, and suggesting the diagnosis of $\mathrm{X}$-Linked Chondrodysplasia Punctata.

Sterol analysis was also performed in blood of the mother and of the maternal grandfather showing sterol ratios of 0.005 and 0.004 , respectively, compatible with CDPX2 in those two individuals.

\section{Molecular diagnosis}

A heterozygous variation was identified by Sanger sequencing in EBP, NM_006579.2:c.301 + 5 G >C (genomic reference sequence NG_007452.1:g.7302 G $>$ C) (Supplementary figure) in the fetal tissue DNA. Familial analysis revealed that the variant was inherited from the mother and was also present as a mosaic variant in the maternal grandfather with an allelic fraction of around $20 \%$ (Fig. 1). Skin biopsies from grandfather confirmed the presence of the variation at mosaic state with a mutated allelic fraction of around $50 \%$, both in normal and affected 
skin. Prediction of an impact on splicing was tested with the splicing prediction module of the Alamut ${ }^{\circledR}$ Visual Software. An abolishment of the wild-type splice site was highlighted by four of the five tools and a significant alteration in the score of the splice site strength was predicted by the latest tool (data not shown).

\section{$\mathrm{X}$ inactivation analysis}

$\mathrm{X}$ inactivation was random in individuals II.2 and III.1, in the blood and fetal cells, respectively.

\section{Splicing functional assay by minigene constructions}

A splicing pCAS2 reporter minigene assay was designed, containing exon 2 of $E B P$ with part of its flanking intronic sequences. After transfection into HeLa cells, splicing for both wild-type and c. $301+5 \mathrm{G}>\mathrm{C}$ mutant constructs were evaluated by RT-PCR analysis and Sanger sequencing. Consistently with the in silico predictions, no transcript retaining exon 2 was detected in presence of the splice variant. In the wild-type context, a predominant band corresponding to normal splicing of the exon 2 was detected. Some minor bands were identified on the electrophoresis gel but were not highlighted in Sanger sequencing, suggesting an artefact from the in vitro model (Fig. 2a).

\section{Tissue mRNA analysis}

The effect of the variant c. $301+5 \mathrm{G}>\mathrm{C}$ was tested using RNA extracted from fetal lung tissue. Gel electrophoresis of RT-PCR showed two bands for fetal tissue (data not shown). The first corresponds to normal splicing whereas the second corresponds to exon 2 skipping (Fig. 2b). The healthy control showed predominantly a band corresponding to normal splicing of EBP (data not shown).

\section{Discussion}

Severe CDPX2 presentations are rare in women. To date, only 15 female cases with prenatal presentation have been reported [7, 15-18].

The propositus was a severely affected female fetus with a variant inherited from her mother presenting only mild CDPX2 symptoms. This post-zygotic nucleotidic change occurred during the pregnancy of the maternal grandfather of the proband (Patient I-1). The grandfather was also mildly affected and presented with phenotypic differences from his siblings. Unlike her mother and grandfather, the fetus showed mostly skeletal features and no skin lesions at the time of termination, while ichthyosis could have been noticed as early as $18 \mathrm{WG}$ [7]. Yet, skin involvement may have developed during the following weeks of pregnancy or even in the postnatal period.

Patient II.2 also had two early miscarriages, suggesting early male lethality of this $E B P$ variant, although other causes cannot be excluded.

Anticipation, phenotypic variation and incomplete penetrance have previously been described as typical features of CDPX2 [11, 15, 19, 20]. Some cases were explained by skewed $X$ inactivation $[19,21]$ although this has not been confirmed in several other family cases [15]. Moreover, the absence of skewed $\mathrm{X}$ inactivation in the studied tissue, does not exclude skewed $\mathrm{X}$ inactivation in the involved cell types such as cartilage or skeletal tissue $[15,22]$.

The c. $301+5 \mathrm{G}>\mathrm{T}$ variant has been previously described in dbSNP as likely benign (rs886038570), but without phenotypic precision. It was predicted to abolish the $3^{\prime}$ splice site of exon 2. Functional studies by RNA analysis and minigene assays consistently found skipping of exon 2 consecutive to the variant. It should be noted that $E B P$ spans 5 exons, four of which are coding with ATG start codon at the beginning of exon 2 , which cause a null allele in this case of abnormal splicing.

Interestingly, this family presented with rather severe skeletal manifestations, as this was the only symptom in the fetus, and both mother and grandfather presented with short stature compared to their unaffected relatives. They had relatively mild cutaneous symptoms, whereas in a previous cohort, eight out of nine fetuses presented with precocious ichthyosis. The pregnancy was terminated earlier than 18 WG for the ninth fetus, at a time when epidermic keratinization had not yet occurred. In the same cohort, seven fetuses out of nine had asymmetric shortening of the long bones, and five had bowing of the long bones [7]. Absence of cutaneous abnormality could be secondary to differential splicing in different target tissues with, for this variant, less exon skipping in the ectodermic tissue and more in mesodermic tissue.

Although CDPX2 is considered lethal in males, some living patients have been described [23]. For most of them, molecular diagnosis had not been performed. BarbozaCerda et al. suggested that clinical variability of CDPX2 in males was part of a continuous phenotypic spectrum [24]. While mutations leading to absent EBP protein can cause intra-uterine lethality in male fetuses, somatic mosaicism [3, 4] or, more rarely, functional $X$ inactivation, such as was described in a 47,XXY patient [5] could cause phenotypic features similar to CDPX2-affected females.

Also, a distinct non-lethal syndrome linked to $E B P$ hypomorphic alleles was described at the hemizygous, nonmosaic state in males [23-26]. Patients present with neurological defects and mental retardation. Arnold et al. speculated that residual isomerase activity allowed survival 
of male fetuses and proposed the name MEND syndrome, for Male $E B P$ disorder with Neurological Defects (MIM \#300960) [27, 28].

We could thus postulate that a complete enzymatic deficit in a cell population caused by a null allele, as seen in CPDX2, may result in the phenotype through accumulation of toxic cholesterol precursors, for example, whereas residual enzymatic activity, such as occurs in MEND syndrome, could cause a neurodevelopmental syndrome without skeleton abnormalities. Interindividual variability in CDPX2 would then be secondary to different degrees of mosaicism (in males and females) or different $\mathrm{X}$ inactivation patterns (in females), or even variable tolerance to sterol precursor toxicity. Considering the numerous genes involved in the cholesterol biosynthesis, undiagnosed variants in related EBP genes may be implicated on the observed variability. However, to our knowledge, no single modifier gene has been identified, suggesting either a heterogeneous, patient-specific mechanism, or, more likely, involvement of variants in several genes in a polygenic model. Unfortunately, because of lack of fetal material, we could not verify this hypothesis by conducting a broader analysis, such as whole exome or whole genome sequencing.

As a conclusion, we described a family carrying an $E B P$ variant leading to a splicing alteration, responsible for CDPX2 in three generations: one mildly affected male carrying the variant at a mosaic state, his daughter who only presented with moderate short stature and xerosis, and a severely affected female fetus. We cannot exclude intrafamilial variability secondary to skewed $\mathrm{X}$ inactivation in target tissues other than the ones studied in mother and child, but it may also be caused by anticipation, as previously postulated. The predominant skeletal phenotype compared with more classical cutaneous features suggests variability in $E B P$ mRNA splicing efficiency in different target tissues.

Acknowledgements The authors thank P. Gaildrat and A. Martins (Inserm U1079, Rouen University, France) for kindly providing the pCAS2 vector. We also wish to thank Jeffrey Arsham, an American medical translator, for rereading this manuscript. We especially thank the family described in this report for her contribution and support.

\section{Compliance with ethical standards}

Conflict of interest The authors declare that they have no conflict of interest.

\section{References}

1. Bruch D, Megahed M, Majewski F, Ruzicka T. Ichthyotic and psoriasiform skin lesions along Blaschko's lines in a woman with X-linked dominant chondrodysplasia punctata. J Am Acad Dermatol. 1995;33:356-60.
2. Posey JE, Burrage LC, Campeau PM, et al. Adult presentation of $\mathrm{X}$-linked Conradi-Hünermann-Happle syndrome. Am J Med Genet A. 2015;167:1309-14.

3. Has C, Bruckner-Tuderman L, Müller D, et al. The Conradi-Hünermann-Happle syndrome (CDPX2) and emopamil binding protein: novel mutations, and somatic and gonadal mosaicism. Hum Mol Genet. 2000;9:1951-5.

4. Aughton DJ, Kelley RI, Metzenberg A, et al. X-linked dominant chondrodysplasia punctata (CDPX2) caused by single gene mosaicism in a male. Am J Med Genet A. 2003;116A:255-60.

5. Sutphen R, Amar MJ, Kousseff BG, Toomey KE. XXY male with $\mathrm{X}$-linked dominant chondrodysplasia punctata (Happle syndrome). Am J Med Genet. 1995;57:489-92.

6. Happle R. X-linked dominant chondrodysplasia punctata. Review of literature and report of a case. Hum Genet. 1979;53:65-73.

7. Lefebvre M, Dufernez F, Bruel AL, et al. Severe X-linked chondrodysplasia punctata in nine new female fetuses. Prenat Diagn. 2015;35:675-84.

8. Leclerc-Mercier S, Dufernez F, Fraitag S, et al. Keratotic follicular plugs with calcifications in Conradi-Hünermann-Happle syndrome: histological, biochemical and genetic testing correlation. Br J Dermatol. 2015;173:1316-8.

9. Silve S, Dupuy PH, Labit-Lebouteiller C, et al. Emopamilbinding protein, a mammalian protein that binds a series of structurally diverse neuroprotective agents, exhibits delta8-delta7 sterol isomerase activity in yeast. J Biol Chem. 1996;271: 22434-40.

10. Braverman N, Lin P, Moebius FF, et al. Mutations in the gene encoding 3 beta-hydroxysteroid-delta 8 , delta 7-isomerase cause $\mathrm{X}$-linked dominant Conradi-Hünermann syndrome. Nat Genet. 1999;3:291-4.

11. Has C, Seedorf U, Kannenberg F, et al. Gas chromatography-mass spectrometry and molecular genetic studies in families with the Conradi-Hünermann-Happle syndrome. J Invest Dermatol. $2002 ; 118: 851-8$.

12. Chevy F, Humbert L, Wolf C. Sterol profiling of amniotic fluid: a routine method for the detection of distal cholesterol synthesis deficit. Prenat Diagn. 2005;25:1000-6.

13. Allen RC, Zoghbi HY, Moseley AB, et al. Methylation of HpaII and HhaI sites near the polymorphic CAG repeat in the human androgen-receptor gene correlates with $\mathrm{X}$ chromosome inactivation. Am J Hum Genet. 1992;51:1229-39.

14. Gaildrat P, Killian A, Martins A, et al. Use of splicing reporter minigene assay to evaluate the effect on splicing of unclassified genetic variants. Methods Mol Biol. 2010;653: 249-57.

15. Hellenbroich Y, Grzeschik KH, Krapp M, et al. Reduced penetrance in a family with $\mathrm{X}$-linked dominant chondrodysplasia punctata. Eur J Med Genet. 2007;50:392-8.

16. Umranikar S, Glanc P, Unger S, et al. X-Linked dominant chondrodysplasia punctata: prenatal diagnosis and autopsy findings. Prenat Diagn. 2006;26:1235-40.

17. Offiah AC, Mansour S, Jeffrey I, et al. Greenberg dysplasia (HEM) and lethal $\mathrm{X}$ linked dominant Conradi-Hünermann chondrodysplasia punctata (CDPX2): presentation of two cases with overlapping phenotype. J Med Genet. 2003;40:e129.

18. Rakheja D, Read CP, Hull D, et al. A severely affected female infant with $\mathrm{x}$-linked dominant chondrodysplasia punctata: a case report and a brief review of the literature. Pediatr Dev Pathol. 2007;10:142-8.

19. Cañueto J, Girós M, Ciria S, et al. Clinical, molecular and biochemical characterization of nine Spanish families with ConradiHünermann-Happle syndrome: new insights into X-linked dominant chondrodysplasia punctata with a comprehensive review of the literature. Br J Dermatol. 2012;166:830-8. 
20. Herman GE, Kelley RI, Pureza V, et al. Characterization of mutations in 22 females with $\mathrm{X}$-linked dominant chondrodysplasia punctata (Happle syndrome). Genet Med. 2002;4:434-8.

21. Shirahama S, Miyahara A, Kitoh H, et al. Skewed X-chromosome inactivation causes intra-familial phenotypic variation of an EBP mutation in a family with X-linked dominant chondrodysplasia punctata. Hum Genet. 2003;112:78-83.

22. Ikegawa $\mathrm{S}$, Ohashi $\mathrm{H}$, Ogata $\mathrm{T}$, et al. Novel and recurrent EBP mutations in X-linked dominant chondrodysplasia punctata. Am J Med Genet. 2000;94:300-5.

23. Bode $\mathrm{H}$, Galm C, Hummler $\mathrm{H}$, et al. Non-lethal non-mosaic male with Conradi-Hunermann syndrome caused by a novel EBP c.356T $>$ G mutation. Am J Med Genet A. 2013;161A:2385-8.

24. Barboza-Cerda MC, Wong LJ, Martínez-de-Villarreal LE, et al. A novel EBP c.224T>A mutation supports the existence of a male-specific disorder independent of CDPX2. Am J Med Genet A. $2014 ; 164 \mathrm{~A}: 1642-7$.
25. Milunsky JM, Maher TA, Metzenberg AB. Molecular, biochemical, and phenotypic analysis of a hemizygous male with a severe atypical phenotype for X-linked dominant ConradiHunermann-Happle syndrome and a mutation in EBP. Am J Med Genet A. 2003;116A:249-54.

26. Furtado LV, Bayrak-Toydemir P, Hulinsky B, et al. A novel Xlinked multiple congenital anomaly syndrome associated with an EBP mutation. Am J Med Genet A. 2010;152A:2838-44.

27. Arnold AW, Bruckner-Tuderman L, Has C, Happle R. Conradi-Hünermann-Happle syndrome in males vs. MEND syndrome (male EBP disorder with neurological defects). Br J Dermatol. 2012;166:1309-13.

28. Hartill VL, Tysoe C, Manning N, et al. An unusual phenotype of $\mathrm{X}$-linked developmental delay and extreme behavioral difficulties associated with a mutation in the EBP gene. Am J Med Genet A. 2014;164A:907-14. 